Gene Expression and Its Discontents 
Rodrick Wallace • Deborah Wallace

\section{Gene Expression and Its Discontents}

The Social Production of Chronic Disease

Second Edition

型 Springer 


\author{
Rodrick Wallace \\ Division of Epidemiology \\ The New York State Psychiatric Institute \\ at Columbia University \\ New York, NY, USA
}

\author{
Deborah Wallace \\ New York, NY, USA
}

ISBN 978-3-319-48077-0

ISBN 978-3-319-48078-7 (eBook)

DOI 10.1007/978-3-319-48078-7

Library of Congress Control Number: 2016955556

(C) Springer International Publishing Switzerland 2010, 2016

This work is subject to copyright. All rights are reserved by the Publisher, whether the whole or part of the material is concerned, specifically the rights of translation, reprinting, reuse of illustrations, recitation, broadcasting, reproduction on microfilms or in any other physical way, and transmission or information storage and retrieval, electronic adaptation, computer software, or by similar or dissimilar methodology now known or hereafter developed.

The use of general descriptive names, registered names, trademarks, service marks, etc. in this publication does not imply, even in the absence of a specific statement, that such names are exempt from the relevant protective laws and regulations and therefore free for general use.

The publisher, the authors and the editors are safe to assume that the advice and information in this book are believed to be true and accurate at the date of publication. Neither the publisher nor the authors or the editors give a warranty, express or implied, with respect to the material contained herein or for any errors or omissions that may have been made.

Printed on acid-free paper

This Springer imprint is published by Springer Nature

The registered company is Springer International Publishing AG

The registered company address is: Gewerbestrasse 11, 6330 Cham, Switzerland 


\section{Preface}

Within the United States, beginning in the late 1980s, several chronic conditions began epidemic increases of both incidence and prevalence. These conditions included asthma, obesity, obesity-related diseases such as diabetes, certain cancers, and Alzheimer's disease (AD). Scientific papers duly reported the existence of genes that putatively made their carriers more vulnerable to these conditions than the general public. However, the genetic composition of US populations did not change so rapidly as to be a basis for these rapid epidemics. HIV/AIDs and tuberculosis, although infectious, are classed as chronic diseases and also became epidemic in the United States during these years. Mental disorders also form an important set of persistent chronic conditions having "environmental" correlations that challenge simple genetic etiologies.

Furthermore, many of these epidemics had geographic foci at multiple levels of organization: neighborhood, municipality, county, state, and country. Many conditions formed "syndemics"-multiple epidemics with the same geography. Poor neighborhoods of color showed heightened incidence and prevalence within cities and formed the centers from which the epidemics spread. Poor neighborhoods that had experienced residential upheavals and instabilities particularly showed great vulnerability. Cities with residential and economic instability showed higher incidence/prevalence than others and formed the foci of spread between metropolitan regions of the country. The states of the Southeast, characterized by extreme social and economic inequality, rigid socioeconomic hierarchy over time, low educational attainment, and a culture-based ideology of individualism, showed the highest incidence/prevalence of obesity, obesity-related diseases and mortalities, AIDS, and AD mortality.

Maps of prevalence and incidence reflect complex cognitive processes. Socioeconomic and environmental signals impinge on populations and their individual constituents, having varying sensitivity and vulnerability. Sensitivity and vulnerability are determined by the interlinked factors of culture, historical trajectory, material resources, social structure and stability, and, most centrally, public policies that can mitigate or exacerbate these factors. 
Individuals and populations are cognitive — able to choose one or a few responses from a larger set of those possible — and act in a multilevel linked process against, or consonant with, these signals. Within individuals, the signals and responses involve internal cognitive processes, for example, the "fight or flight" response to a perceived threat, a response involving the brain, pituitary gland, adrenal gland, circulatory system, and energy regulatory mechanisms. Epigenetic changes triggered by the signals or the initial recognition of the signals induce cognitive genetic processes as well.

The strong influence of cognitive processes at levels of organization from the nation down to the molecular biology of individuals implies that information theory, control theory, and mathematical modeling based on them can describe and predict patterns of chronic disease in cultural and socioeconomic contexts. In this book, we develop statistical models of such processes that are akin in spirit to-but different from-ordinary regression models and report empirical data and analyses to illustrate this paradigm.

The first six chapters use the asymptotic limit theorems of information theory to understand how epigenetic context affects organismal development by invoking a cognitive paradigm for gene expression. A simple argument suggests that epigenetic information sources act as analogs to a tunable catalyst, directing development to different characteristic pathways in a manner similar to ecosystem resilience shifts. The results have significant implications for epigenetic epidemiology, showing how environmental stressors, in a large sense, can induce a spectrum of chronic disorders.

Chapter 7 examines the US obesity epidemic from this viewpoint. Chapters 8 and 9 apply the perspective to heart disease and cancer and, in their later sections, introduce tools from control theory that illuminate the central role of "environmental" context. Chapters 10-12 apply the general model to a number of diseases broadly associated with obesity that are becoming pandemic, using US data at different scales of observation. Chapters 13 and 14 study how "culture" and "environment" affect the onset and progression of mental disorders. Chapters 15-18 provide case histories involving low weight births, "Right-to-work" laws and AD, diabetes and thyroid cancer in Manhattan's Chinatown, and the etiology of violence and obesity. Chapter 19 explores a composite model of psychopathology, sleep, and culture in the context of environmental insult.

The theory appears to work very well at individual and simple aggregate levels for a number of chronic conditions in stressed populations. Environments that can be characterized as having regularities of "grammar" and "syntax" can interact with organismal development via epigenetic catalysis to literally write distorted images of themselves onto the human life course in a highly pleiotropic and often punctuated manner, producing trajectories to a range of serious dysfunctions. Communities, in which individuals respond collectively, however, display more complicated patterns of chronic disease that may represent another example of a "mesoscale resonance" in which the dynamics of ecological keystone structures entrain phenomena at other scales.

One implication of this work is that pandemic chronic diseases at both the individual, via pleiotropy, and the population scales, via collective modalities 
of gene expression, are unlikely to respond to individual-level-i.e., medicalinterventions in the face of serious, persistent individual and community stress and may require large-scale changes in public policy and resource allocation for their amelioration. Drugs powerful enough to affect deleterious epigenetic programming are likely to trigger profound iatrogenic "side effects" that, over the life course, would not only obviate the intervention but most likely lead to shortened life spans.

The book, a synthesis across a number of peer-reviewed publications and additional material written for this volume, can be read at several levels. Chapters 1 and 6-20 form a natural introductory unit that can be followed by the more mathematical sections as desired. Mathematical details are collected in an appendix. However, the control theory methods introduced in the latter parts of Chaps. 8 and 9 are used extensively in Chaps. 14 and 19.

In sum, we provide something of a badly needed corrective to simplistic "genetic" explanations of chronic disease that, politically, are inherently useful for blaming the victim and undercutting intervention strategies seen as disruptive to the interests of ruling elites. We focus instead on the generation of disease phenotypes by social forces that can be mitigated or amplified by public policy. 


\section{Contents}

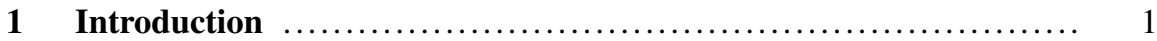

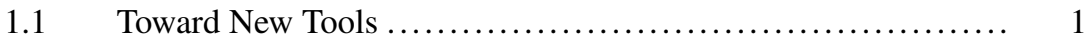

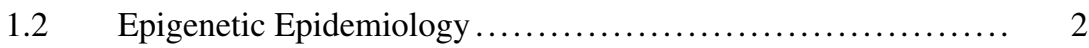

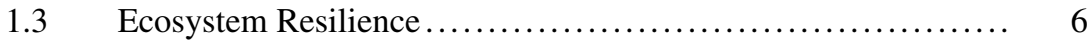

1.3.1 Comorbid Disorders............................ 7

1.3.2 Some Cognitive Modules of Human Biology ........... 8

1.3.3 Cognition as "Language": an Introduction............ 11

1.3.4 A Multiplicity of Resilience Topologies .............. 12

1.3.5 In Summary.................................. 14

1.4 A First Survey of the Obesity Pandemic in the USA............ 15

2 Models of Development ....................................... 27

2.1 The Spinglass Model ............................... 27

2.2 Shifting Perspective: Cognition as an Information Source....... 30

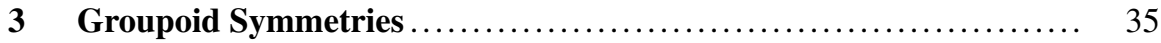

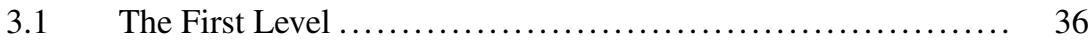

3.2 The Second Level .................................. 36

3.3 Spontaneous Symmetry Breaking ........................ 37





4.1 The Basic Idea .................................... 43

$4.2 \quad$ Rate Distortion Dynamics ............................. 44

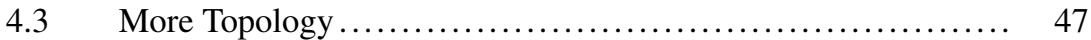

$4.4 \quad$ Inherited Epigenetic Memory ............................ 48

$4.5 \quad$ Multiple Processes ................................... 49

$4.6 \quad$ "Coevolutionary" Development ........................ 50

$4.7 \quad$ Multiple Models ....................................... 51

$4.8 \quad$ Epigenetic Focus .................................... 52 


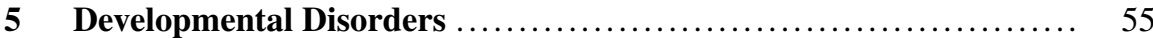

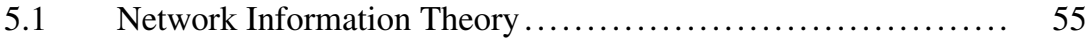

5.2 Embedding Ecosystems as Information Sources ............. 56

$5.3 \quad$ Ecosystems Farm Development .......................... 56

$5.4 \quad$ A Simple Probability Argument............................. 59

5.5 Developmental Shadows.................................. 60

5.6 Epigenetic Programming of Artificial Systems



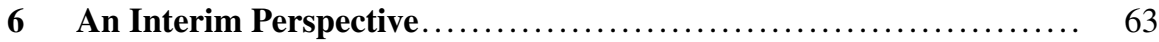

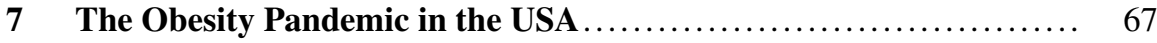

$7.1 \quad$ Introduction ............................................ 67

7.2 Stress and the HPA Axis................................. 68

$7.3 \quad$ HPA Axis Cognition ................................... 69

7.4 Interacting Information Sources........................... 69

$7.5 \quad$ A HPA Axis Model ...................................... 71

7.6 Psychosocial Stress, Occupational Control, and Weight Gain .... 74

7.7 Obesity as a Developmental Disorder...................... 74

7.8 Recent Trajectories of Structured Stress in the USA ............ 75

$7.9 \quad$ Confronting the Obesity Epidemic ...................... 82

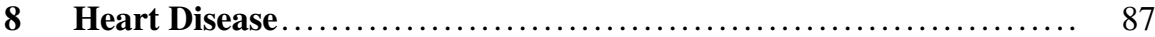

$8.1 \quad$ Coronary Heart Disease in the USA ..................... 87

8.1.1 Cognition, Immune Cognition, and Culture ........... 90

8.1.2 Punctuated Interpenetration: Adapting to Pathogenic Hierarchy .............................. 93

8.1.3 Implications for Intervention ...................... 94

$8.2 \quad$ Thrombogenesis........................................ 97

8.2.1 Control Theory: The Data Rate Theorem ............. 98

8.2.2 The Information Cost of Hemostatic Regulation ....... 99

8.2.3 Control Failure Dynamics .......................... 101

8.2.4 Cognitive Symmetry Breaking ..................... 102

8.2.5 Onset of "Turbulent" Clot Growth ................... 103

8.2.6 Implications for Intervention .................... 104



9.1 A Developmental Model.............................. 107

9.2 Environmental Insult and Regulatory Failure ............... 114





10.2 Nonorthogonal Eigenmodes of Immune Cognition ............. 124

10.3 Circadian and Other Cycles ............................ 126

10.4 The Retina of the Immune Response ....................... 129

10.5 Circadian-Hormonal Cycle Synergism ................... 130

10.6 The Cognitive HPA Axis ............................... 131 
10.7 Phase Transitions of Interacting Information Systems ......... 132

10.8 Autoimmune Disease ................................ 133

10.9 An Application to Asthma............................. 135

10.10 Images of Pathogenic Social Hierarchy ................... 136

11 Demoralization and Obesity in Upper Manhattan............... 141

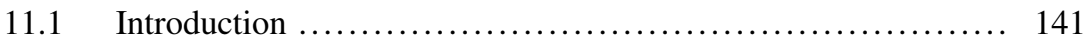

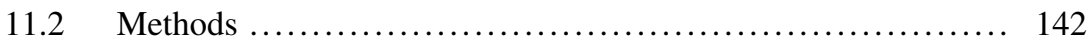

11.2.1 Recruitment of the Mothers....................... 142

11.2.2 Prenatal Questionnaire ............................ 142

11.2.3 Socioeconomic Data .............................. 144

11.2.4 Chronic Community Stress Index ................... 144

11.3 Data Analysis .................................... 147

11.4 Modeling Demoralization and Chronic Stress ................ 147

11.5 Results ......................................... 148

11.5.1 Demoralization and Chronic Stress ................. 148

11.5.2 Signal Transduction Modeling....................... 150

11.5.3 Body Mass Index ................................. 151

11.5.4 Household Deprivation $. . \ldots \ldots \ldots \ldots \ldots \ldots \ldots \ldots \ldots . \ldots \ldots 2$

11.6 Discussion .............................................. 154

11.6.1 The Asymmetric Inverted "U" Curve ................ 154

11.6.2 The Body mass Index ........................... 155

11.7 Conclusion ............................................... 157

12 Death at an Early Age: AIDS and Related Mortality

in New York City ........................................... 161

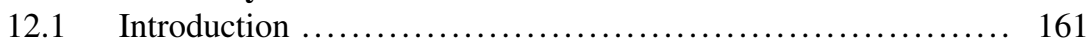

12.2 Data and Analysis ................................... 163

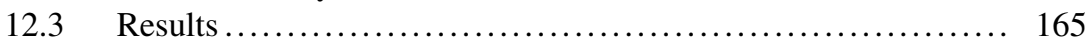

12.4 Discussion ............................................. 170

13 Western Atomism and Its Culture-Bound Syndromes ............. 181

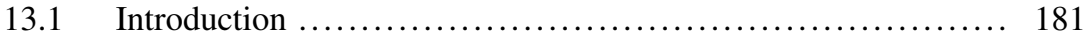

13.2 Western Atomistic Economics ............................ 183

13.3 Cognition as an Information Source ....................... 185

13.4 Environment as an Information Source ...................... 187

13.5 Interacting Information Sources....................... 188

13.6 Crosstalk Topologies.................................. 189

13.7 Punctuated Critical Phenomena ......................... 190



14 Environmental Induction of Neurodevelopmental Disorders........ 195

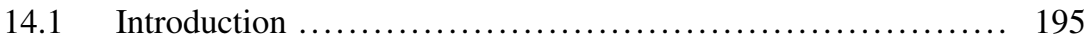

14.2 Stabilizing Gene Expression ........................... 197

14.3 The Cost of Regulation .................................. 198



14.5 The Dynamics of Control Failure ........................ 199 
14.6 Failure of Regulation as a Phase Transition ................ 201

14.7 Estimating the Quadratic Variation ....................... 203

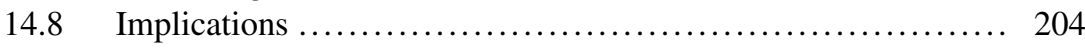

15 Mass De-Housing and Low-Weight Births................... 207



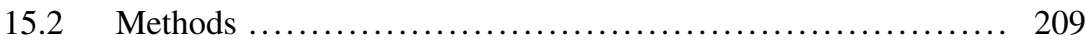

$15.3 \quad$ Results ......................................... 210

15.3.1 Upper Manhattan Birth Cohort .................... 210

15.4 The Changing Geography of Low Birth-Weight

Incidence 1990-2008 .............................. 210

15.4.1 Maps ..................................... 210

15.4.2 Regressions ................................. 211

15.5 Discussion ......................................... 215

15.5.1 Center Data ................................. 217

15.5.2 What the Health District Maps Show ............... 218

15.5.3 What the Regressions Show ..................... 219



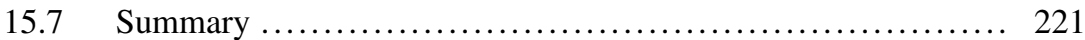

16 Alzheimer's Disease and "Right-to-Work" Laws ................ 223



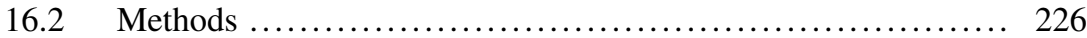

16.3 Results ................................................. 227

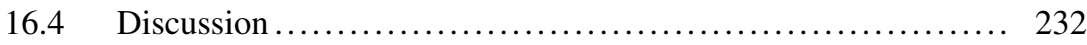

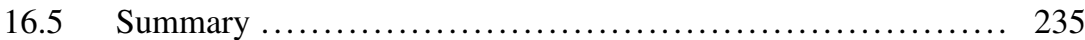

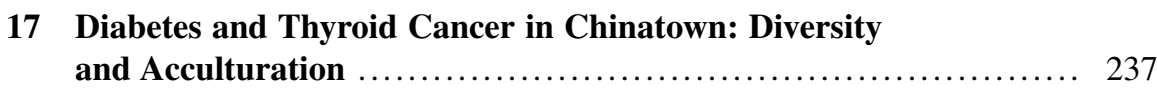





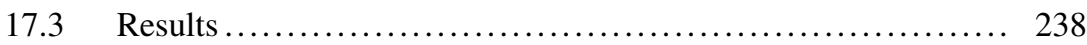

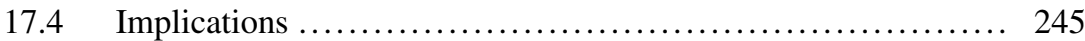

17.4.1 Limitations of This Study ....................... 247

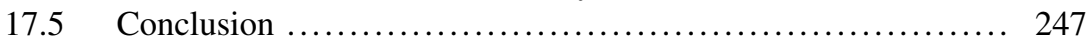

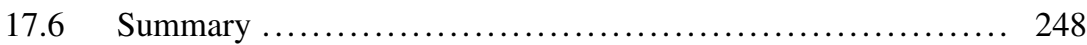

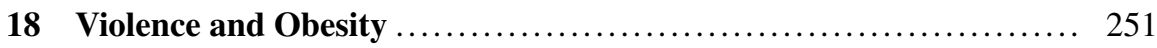



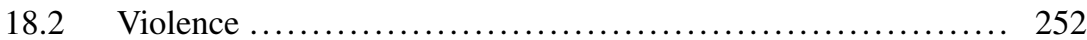

18.3 Organic Pollutants and Obesity/Diabetes ................... 261

18.4 Summary and Conclusion .......................... 265

19 Psychopathology, Sleep, and Culture ....................... 269

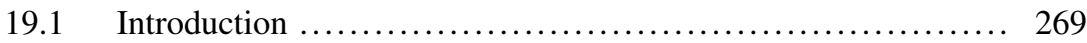

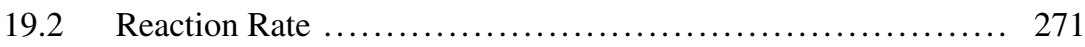

19.3 The Data Rate Theorem ................................ 274 
19.4 Elementary Rate Distortion Dynamics.................. 275

19.5 An Optimization Model ............................... 277

19.6 Transition Dynamics ............................... 280

19.7 Environmental Induction of Sleep Disorders ............... 281

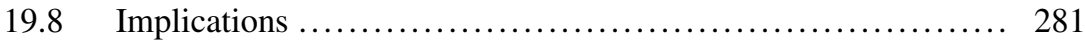



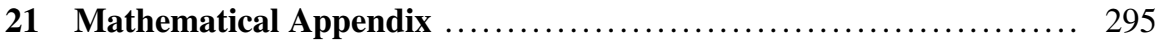

21.1 The Shannon Coding Theorem........................ 295

21.2 The "Tuning Theorem" ............................... 297

21.3 The Shannon-McMillan Theorem ....................... 299

21.4 The Rate Distortion Theorem ........................... 301

21.5 Groupoids ...................................... 303

21.5.1 Basic Ideas................................. 303

21.5.2 Global and Local Symmetry Groupoids ............. 305

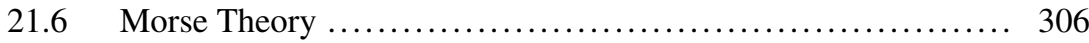

21.7 Generalized Onsager Theory $\ldots \ldots \ldots \ldots \ldots \ldots \ldots \ldots \ldots \ldots \ldots . \ldots \ldots$

21.8 Black-Scholes Calculation ........................... 310

21.9 Large-Scale Noise-Driven Cognitive Switch ................ 311

21.10 The Data Rate Theorem ............................... 312

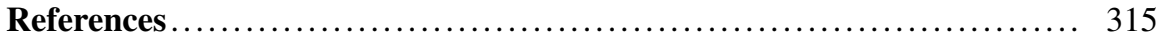



